\title{
Tratamiento del carcinoma diseminado de estómago: ¿pauta estándar?
}

\author{
C. García-Girón, E. Lastra Aras, R. Hernández García
}

El $80-90 \%$ de los pacientes con cáncer gástrico presentan enfermedad diseminada en algún período de su evolución, bien en el momento del diagnóstico como estadio IV (40\%) o bien como enfermedad recurrente (70\%), a consecuencia de la recaída del tratamiento quirúrgico curativo de los estadios I-III.

El tratamiento con quimioterapia del cáncer gástrico diseminado es eminentemente paliativo. Su beneficio debe medirse en parámetros de supervivencia, de calidad de vida o de alivio sintomático, de toxicidad y de coste económico.

Cuatro ensayos aleatorizados han evaluado el beneficio en supervivencia de la quimioterapia frente al tratamiento de soporte o sintomático solos, en cáncer gástrico diseminado. Globalmente, la quimioterapia retrasa la mediana del tiempo a la progresión en 3-4 meses y prolonga la mediana de supervivencia en 3-7 meses ${ }^{1-4}$ (Tabla I).

La administración de la quimioterapia de forma inmediata, tras el diagnóstico del cáncer gástrico diseminado, frente a su retraso a demanda de los síntomas no controlables con tratamiento sintomático solo, supone un aumento de la mediana de supervivencia ( 10 meses versus 4 meses) y una mayor proporción de pacientes con mejoría de la calidad de vida $(75 \%$ versus $25 \%)$, según un pequeño estudio aleatorizado de Glimelius et al ${ }^{5}$. Esto pone de manifiesto que la ausencia o escasez de síntomas y por tanto un mejor estado funcional, se sigue de un mayor beneficio en supervivencia y calidad de vida para los pacientes con cáncer gástrico diseminado.

El estudio aleatorizado de Glimelius et al., que compara quimioterapia (Etopósido-Ácido folínico-Fluorouracilo -ELF-, - Ácido folínico-Fluorouracilo en pacientes con estado funcional con índice de Karnofsky (70 y edad mayor de 60 años) frente a tratamiento de soporte en 61 pacientes con cáncer gástrico diseminado, muestra, además de un aumento de la supervivencia, una mejoría de la calidad de vida durante al menos 4 meses en el $45 \%$ de los pacientes que se

Servicio de Oncología Médica Hospital General Yagüe Burgos tratan con quimioterapia, frente a sólo un $20 \%$ en los que reciben tratamiento sintomático $(p<0,05)^{4}$.

En este contexto de discreto beneficio importa el coste económico de la quimioterapia. En un estudio realizado en la Universidad de Upsala sobre el coste-eficacia de la quimioterapia paliativa en el cáncer gastrointestinal avanzado, los autores encuentran que el tratamiento con quimioterapia aumenta el gasto en un 50\%; pero el aumento de supervivencia y de calidad de vida que la quimioterapia produce compensa su incremento en el gasto, de forma que el tratamiento citostático del cáncer gástrico diseminado es coste-eficaz y coste-útil ${ }^{6}$

En conclusión, la quimioterapia aporta una mayor supervivencia y una mejor calidad de vida a los pacientes con cáncer gástrico diseminado. Aunque este beneficio es discreto, justifica el incremento de gasto de la administración de fármacos citostáticos. Por tanto, la quimioterapia es el tratamiento de elección en los pacientes con cáncer gástrico avanzado con buen estado general.

Prácticamente todos los citostáticos se han estudiado en ensayos de fase II en el cáncer gástrico diseminado. Sólo una pequeña proporción de ellos han demostrado una respuesta objetiva (15\%), necesaria para ser considerados activos en monoterapia. Obviamente, la duración de la respuesta es menor de 6 meses, la tasa de respuestas completas es menor del $5 \%$ y la repercusión en la supervivencia global es escasa (Tabla II).

Clásicamente, el Fluorouracilo es el citostático más utilizado y cuya actividad en monoterapia es más consistente. Aunque no hay estudios que comparen la administración en bolo frente a la administración en infusión continua de Fluorouracilo, es de prever que su administración en infusión continua, así como su modulación con ácido folínico sean más activas y así se han incorporado en combinaciones con Cisplatino. Entre los análogos orales de Fuorouracilo destaca el S-1, compuesto de Tegafur con dos moduladores (5-cloro2,4-dihidroxipiridina y oxonato potásico), que en dos ensayos de fase II muestra una respuesta objetiva media del $45 \% 7$.

La combinación de dos o tres citostáticos activos es el paso lógico para abordar la heterogeneidad celular y la multirresistencia del cáncer gástrico diseminado. Estas combinaciones muestran una media de respuestas objetivas del 30$50 \%$ y de medianas de supervivencia de 7-9 meses. Corresponden fundamentalmente a ensayos de fase II con un inter- 
TABLA I

Ensayos de quimioterapia frente a tratamiento sintomático en cáncer gástrico diseminado

\begin{tabular}{|c|c|c|c|c|c|c|}
\hline $\begin{array}{l}\text { Autor, año } \\
\text { Referencia }\end{array}$ & Tratamiento & № Pacientes & Respuesta Objetiva (\%) & $\begin{array}{l}\text { Progresión de } \\
\text { enfermedad (\%) }\end{array}$ & $\begin{array}{c}\text { Tiempo mediano } \\
\text { a la progresión (meses) }\end{array}$ & $\begin{array}{c}\text { Supervivencia } \\
\text { mediana (meses) }\end{array}$ \\
\hline Pyrhönen, & FEMTX & 17 & 29 & 24 & $5,4^{*}$ & $12,3^{*}$ \\
\hline $1995^{1}$ & Sintomático & 19 & - & 80 & 1,7 & 3,1 \\
\hline Murad, & FAMTX & 30 & 50 & - & - & $10^{*}$ \\
\hline $1993^{2}$ & Sintomático & 10 & - & - & - & 3 \\
\hline Scheithaver, & EpilF & 18 & 38 & 4 & $>5^{*}$ & $>7,5^{*}$ \\
\hline $1995^{3}$ & Sintomático & 19 & - & 53 & 2 & 4 \\
\hline Glimelius, & ELF o LVFU & 31 & 23 & 30 & $6^{*}$ & $8^{*}$ \\
\hline 19974 & Sintomático & 30 & - & - & 2 & - \\
\hline
\end{tabular}

FEMTX: Fluorouracilo-Epiadriamicina-Metotrexate. FAMTX: Fluorouracilo-Adriamicina-Metotrexate. EpiLF: Epiadriamicina-Ácido FolínicoFluorouracilo.ELF: Etoposido-Ácido Folínico-Fluorouracilo. FULV: Ácido Folínico-Fluorouracilo. * Significación estadística.

TABLA ॥ Citostáticos más activos en monoterapia en cáncer gástrico
diseminado

\begin{tabular}{lcc}
\hline Citostático & № pacientes & Respuesta objetiva (\%) \\
\hline FLUOROURACILO & 416 & 21 \\
UFT & 188 & 28 \\
S-1 & 101 & 45 \\
MITOMICINA & 211 & 30 \\
ADRIAMICINA & 141 & 17 \\
EPIADRIAMICINA & 80 & 19 \\
BCNU & 23 & 17 \\
CISPLATINO & 139 & 19 \\
ETOPOSIDO (VO) & 14 & 21 \\
DOCETAXEL & 157 & 22 \\
PACLITAXEL & 82 & 15 \\
IRINOTECAN (CPT-11) & 66 & 23 \\
\hline
\end{tabular}

valo amplio en los resultados, según los sesgos de selección de este tipo de estudios (Tabla III). Una característica común de estos regímenes es su asociación basada en la actividad de Fluorouracilo y/o Cisplatino, de forma que su probada sinergia constituye una combinación de referencia (FUP) y origen de otros regímenes de éxito como FAP y ECF.

La modulación de Fluorouracilo por Metotrexate (FAMTX) ha dado paso la modulación por ácido folínico (ELF) y a la administración en infusión continua (FUP) o prolongada (ECF).

La actividad en monoterapia de Docetaxel e Irinotecan muestran unos resultados alentadores en combinación con cisplatino en dos ensayos de fase II.

Los ensayos de fase III entre las distintas combinaciones ponen de manifiesto los siguientes resultados ${ }^{\text {8-19 }}$ (Tablas $\mathrm{IVa}, \mathrm{IVb}$ y IVc).

1) La superioridad en tasa de respuestas objetivas de las combinaciones con Cisplatino-Fluorouracilo frente a los regímenes sin Cisplatino 9, 10, 12, 13, 15, 19 .

2) Este incremento de respuestas objetivas no se traduce en un aumento de la supervivencia global, cuya mediana se mantiene en 7-9 meses. Hace excepción el estudio de Webb el al, en que el régimen ECF muestra una significativa mayor respuesta objetiva y supervivencia que la combinación FAMTX'12.

3) La combinación de Irinotecan-Fluorouracilo muestra una elevada respuesta objetiva y una prolongada supervivencia, frente a combinaciones con Cisplatino, en dos de los ensayos en fase III17, 19 .

TABLA III

Combinaciones de citostáticos más activas en monoterapia en cáncer gástrico diseminado

\begin{tabular}{lccc}
\hline Combinación & No Pacientes & Respuesta Objetiva (\%) & Supervivencia mediana (meses) \\
\hline Fluorouracilo-Adriamicina-Mitomicina (FAM) & 656 & 30 & $6-9$ \\
Fluorouracilo-Adriamicina-Metotrexate (FAMTX) & 364 & 41 & $3,5-10,5$ \\
Etoposido-Ácido Folínico-Fluorouracilo (ELF) & 183 & 21 & $7,2-11$ \\
Fluorouracilo-Cisplatino (FUP) & 275 & 36 & $7-10,6$ \\
Fluorouracilo-Adriamicina-Cisplatino (FAP) & 234 & 34 & $6-13$ \\
Etoposido-Adriamicina-Cisplatino (EAP) & 173 & 53 & $6-9$ \\
Fluorouracilo-Epiadriamicina-Cisplatino (ECF) & 416 & 43,5 & $8,7-9,4$ \\
Taxotere-Cisplatino (TP) & 48 & 56 & 9 \\
Irinotecan-Cisplatino (IP) & 44 & 48 & 10,1 \\
\hline
\end{tabular}


TABLA IVa

Ensayos de fase III de las combinaciones de citostáticos más activas en monoterapia en cáncer gástrico diseminado

\begin{tabular}{llccc}
\hline $\begin{array}{l}\text { Autor, año } \\
\text { Referencia }\end{array}$ & Tratamiento & No Pacientes & Respuesta Objetiva (\%) & Supervivencia mediana (meses) \\
\hline Wils, & FAM & 105 & 9 & 4 \\
$1991^{8}$ & FAMTX & 107 & $41^{*}$ & $6^{*}$ \\
Coconni, & FAM & 52 & 15 & 5,6 \\
$1994^{9}$ & PELF & 85 & $43^{*}$ & 8,1 \\
Kim, & FU & 94 & 26 & 7 \\
1993 10 & FAM & 103 & 25 & 6,8 \\
Kelsen, & FUP & 103 & $51^{*}$ & 8,6 \\
$1992{ }^{11}$ & FAMTX & 30 & 33 & 7,3 \\
Webb, & EAP & 30 & 20 & 6,1 \\
199712 & FAMTX & 130 & 21 & 6,1 \\
\hline
\end{tabular}

FAM: Fluorouracilo-Adriamicina-Mitomicina. FAMTX: Fluorouracilo-Adriamicina-Metotrexate. PELF: Cisplatino-Epiadriamicina-Ácido Folínico-Fluorouracilo. FU: Fluorouracilo. FUP: Fluorouracilo-Cisplatino. EAP: Etoposido-Adriamicina-Cisplatino. ECF: Fluorouracilo-Epiadriamicina-Cisplatino. ${ }^{*}$ Significación estadística.

\section{TABLA IVb}

Ensayos de fase III de las combinaciones de citostáticos más activas en monoterapia en cáncer gástrico diseminado

\begin{tabular}{llccc}
\hline $\begin{array}{l}\text { Autor, año } \\
\text { Referencia }\end{array}$ & Tratamiento & No Pacientes & Respuesta Objetiva (\%) & Supervivencia mediana (meses) \\
\hline Vanhoefer, & FUP & 134 & 20 & 7,2 \\
$2000^{13}$ & ELF & 132 & 9 & 7,2 \\
& FAMTX & 133 & 12 & 6,7 \\
Cullinan, & FU & & & 6,1 \\
$1994^{14}$ & FAP & 256 & & NA \\
& FAMe & & 11,4 & 6,1 \\
Ohtzu, & FAMe-Triazinate & 105 & $34,3^{*}$ & 7,7 \\
$2003{ }^{15}$ & FU & 105 & 8,6 & 7,1 \\
& FUP & 70 & & 6,3 \\
\hline
\end{tabular}

FAMTX: Fluorouracilo-Adriamicina-Metotrexate. ELF: Etoposido-Ácido Folínico-Fluorouracilo. FAMe: Fluorouracilo-Adriamicina-Metil-CCNU. FU: Fluorouracilo. FUP: Fluorouracilo-Cisplatino. FAP: Fluorouracilo-Adriamicina-Cisplatino. UFT-MMC: UFT-Mitomicina C. * Significación estadística.

TABLA IVc

Ensayos de fase III de las combinaciones de citostáticos más activas en monoterapia en cáncer gástrico diseminado

\section{Autor, año}

Referencia

Tratamiento № Pacientes

Respuesta Objetiva (\%)

Supervivencia mediana (meses)

\begin{tabular}{llccc}
\hline Ross, & & 290 & 42,4 & 9,4 \\
$2002{ }^{16}$ & ECF & 290 & 44,1 & 8,7 \\
Pozzo, & MCF & 74 & 33,8 & 10,7 \\
$2001{ }^{17}$ & FULV-CPT & 72 & 27,8 & 6,5 \\
Ajani, & P-CPT & 76 & 23,2 & 8,5 \\
$2003{ }^{18}$ & FUP & 79 & $38,7^{*}$ & $10,2^{*}$ \\
Rougier, & TXT-FUP & 45 & 13 & 6,8 \\
$2003{ }^{19}$ & FULV & 44 & 27 & 9,5 \\
& FULV-P & 45 & 40 & 11
\end{tabular}

FUP: Fluorouracilo-Cisplatino. ECF: Fluorouracilo-Epiadriamicina-Cisplatino. MCF: Mitomicina-Epiadriamicina-Cisplatino. FULV-CPT: Ácido Folínico-Fluorouracilo-Irinotecan. P-CPT: Cisplatino-Irinotecan. TXT-FUP: Taxotere-Fluorouracilo-Cisplatino. FULV: Ácido Folínico-Fluorouracilo. FULV-P: Ácido FolínicoFluorouracilo-Cisplatino. * Significación estadística. 


\section{García-Girón y cols.}

4) La adición de Docetaxel a la combinación de Cisplatino-Fluorouracilo produce un significativo aumento de la tasa de respuestas objetivas y de la supervivencia en el estudio de Ajani et al. ${ }^{18}$.

Estos datos muestran que el panorama de la quimioterapia del cáncer gástrico diseminado apenas ha cambiado en los ultimos 25 años: $30-50 \%$ de respuesta objetiva y 7-9 meses de mediana de supervivencia; los resultados superiores se deben más a factores pronósticos de la enfermedad o del paciente que a la eficacia de la quimioterapia.

La adecuación de este hecho a la intención paliativa de la quimioterapia del cáncer gástrico diseminado en forma de tratamiento estándar se debe apoyar nuevamente en los parámetros de beneficio: supervivencia, calidad de vida, toxicidad y coste económico.

En este sentido, la combinación Cisplatino $\left(100 \mathrm{mg} / \mathrm{m}^{2}\right.$ día 1, cada 3 semanas)-Fluorouracilo $\left(1000 \mathrm{mg} / \mathrm{m}^{2}\right.$ ic, días $1-5$, cada 3 semanas) es el régimen más consolidado y que mejor se adecúa al beneficio de los pacientes con cáncer gástrico diseminado cuyo buen estado general (PS ( 2) y funcionalidad orgánica prevén un aceptable perfil de tolerancia. En pacientes ancianos o con deterioro de funciones orgánicas que limiten la tolerancia a Cisplatino, se debe utilizar combinaciones también activas como ELF, FULV, FULV-CPT.

\section{Bibliografía}

1. Pyrhönen S, Kuitunen T, Nyandoto P, Kouri M. Randomized comparison of fluouracil, epidoxorubicin and methotrexate (FEMTX) plus supportive care with supportive care alone in patients with non-resectable gastric cancer. $\mathrm{Br} J$ Cancer 1995; 71:587-91.

2. Murad AM, Santiago FF, Petroianu A, et al. Modified therapy with 5-fluorouracil, doxorubicin and methotrexate in advanced gastric cancer. Cancer 1993; 72:31-41.

3. Scheithauer W, Kornek G, Zeh B, et al. Palliative chemotherapy versus supportive care in patients with metastatic gastric cancer: a randomized trial. Second international conference on biology, prevention and treatment of gastrointestinal malignancies. Colonia (Alemania) 1995; 68.

4. Glimelius B, Ekström K, Hoffman K, Graf W, et al. Randomized comparison between chemotherapy plus best supportive care with best supportive care in advanced gastric cancer. Ann Oncol 1997; 8:163-8

5. Glimelius B, Hoffman K, Haglund U, Nyren O, Siöden PO. Initial or delayed chemotherapy with best supportive care in advanced gastric cancer. Ann Oncol 1994; 5:189-90.

6. Glimelius B, Hoffman K, Graf W, et al. Cost-effectiveness of palliative chemotherapy in advanced gastrointestinal cancer. Ann Oncol 1995; 6:267-74

7. Ohtsu A, Boku N, Sakata et al. S-1 is highly active for patients with advanced gastric cancer. Second international conference on biology, prevention and treatment of gastrointestinal malignancies. Colonia (Alemania) 1998; 50.

8. Wils JA, Klein HO, Wagener DJ, et al. Sequential high-dose methotrexate and fluorouracil combined with doxorubicin a step ahead in the treatment of advanced gastric cancer: a trial of the European Organization for Research and Treatment of Cancer Gastrointestinal Tract Cooperative Group. J Clin Oncol $1991 ; 9: 827-31$
9. Cocconi $G$, Bella $M$, Zironi $S$, et al. Fluorouracil, doxorubicin, and mitomycin combination versus PELF chemotherapy in advanced gastric cancer: a prospective randomised trial of the ltalian Oncology Group for Clinical Research. J Clin Oncol 1994; 12:2687-93.

10. Kim NK, Park YS, Heo DS, et al. A phase III randomised study of 5-fluorouracil and cisplatin versus 5-fluorouracil, doxorubicin and mitomycin $\mathrm{C}$ versus 5 -fluorouracil alone in the treatment of advanced gastric cancer. Cancer 1993; 71:3813-8.

11. Kelsen D, Atiq OT, Saltz L, et al. FAMTX versus etoposide, doxorubicin, and cisplatin: a random assignment trial in gastric cancer. J Clin Oncol 1992; 10:541-8.

12. Vanhoefer $U$, Rougier $P$, Wilke $H$, et al. Final Results of a randomized phase III trial of sequential high-dose Methotrexate, Fluorouracil, and Doxorubicin versus Etoposide, Leucovorin, and Fluorouracil versus Infusional Fluorouracil and Cisplatin in advanced gastric cancer: A trial of the European Organization for Research and Treatment of Cancer Gastrointestinal Tract Cancer Cooperative Group. J Clin Oncol 2000; 18:2648-57.

13. Webb A, Cunningham $D$, Scarffe $\mathrm{JH}$, et al. Randomized trial comparing epirubicin, cisplatin and fluorouracil versus fluorouracil, doxorubicin and methotrexate in advanced esophagogastric cancer. J Clin Oncol 1997; 15:261-7.

14. Cullinan SA, Moertel CG, Wieand HS, et al. Controlled evaluation of three drug combination regimens versus fluorouracil alone for the therapy of advanced gastric cancer. North Central Cancer Treatment Group. J Clin Oncol 1994; 12:412-6.

15. Ohtsu A, Shimada Y, Shirao K, et al. Randomized phase III trial of Fluorouracil alone versus Fluorouracil plus Cisplatin versus Uracil and Tegafur plus Mitomycin in patients with unresectable, advanced Gastric Cancer: The Japan Clinical Oncology Group Study (JCOG9205) J Clin Oncol 2003; 21:54-9.

16. Ross $P$, Nicolson $M$, Cunningham $D$, et al. Prospective randomized trial comparing Mitomycin, Cisplatin, and protracted venous-infusion Fluorouracil (PVI 5-FU) with Epirubicin, Cisplatin, and PVI 5-FU in advanced esophagogastric Cancer. J Clin Oncol 2002; 20:1996-2004.

17. Pozzo $C$, Bugat $R$, Peschel $C$, et al. Irinotecan in combination with CDDP or 5-FU and Folinic Acid is active in patients with advanced gastric or gastro-oesophageal junction adenocarcinoma: Final results of a randomized phase II study. Proc Am Soc Clin Oncol 2001; 20:134a (abstr 531).

18. Ajani JA, Van Cutsem E, Moiseyenko V, et al. Docetaxel (D), cisplatin, 5-fluorouracil compare to cisplatin (C) and 5-fluorouracil $(F)$ for chemotherapy-naïve patients with metastatic or locally recurrent, unresectable gastric carcinoma (MGC): Interim results of a randomized phase III trial (V325). Proc Am Soc Clin Oncol 2003; 22: 249, (abstr 999).

19. Rougier $P$, on behalf FFCD Gastric Cancer Study 9803. Final results of FFCD study in metastaticgastric cancer with Campto/LV5FU2. Second gastro-intestinal clinical update meeting. Tenerife, Spain, 11 october 2003.
Correspondencia:

Dr. C. García-Girón

Servicio de Oncología Médica

Hospital General Yagüe

Avenida del Cid, 96

E-09005 Burgos.

E-mail: giron@hgy.es 